\title{
A Cross-sectional Study on the Follicular Fluid Concentration of Some Interleukins and Clinical Factors in Polycystic Ovary Syndrome Patients
}

\author{
Mohammad Ghodsi $^{1 \oplus}$, Vida Hojati $^{1^{*}(\mathbb{D}}$, Armin Attaranzade $^{2}{ }^{(}$, Bita Saifi $^{3}$ \\ Abstract \\ Objectives: Cytokines regulate ovarian activity through controlling internal ovarian processes as paracrine or autocrine regulators. \\ In the current study, the follicular fluid (FF) concentration of some cytokines including interleukin (IL-3), IL-5, and IL-6 were \\ investigated and compared between 39 patients diagnosed with polycystic ovary syndrome (PCOS) and 34 healthy normal women \\ with male factor infertility as the control group. \\ Materials and Methods: To this end, FF samples were collected by following gonadotropin-releasing hormone antagonist protocol. \\ Then, the FF concentration of the studied cytokines was evaluated using the enzyme-linked immunosorbent assay method. Finally, \\ the characteristics of the participants were collected and compared between PCOS and control groups. \\ Results: Based on the results, the FF concentration of IL-3 and IL-5 revealed a remarkable reduction in the PCOS group in \\ comparison to the control group ( $P=0.0004$ and $P=0.04$, respectively). In addition, there were noticeable differences in the body \\ mass index $(P=0.009)$, endometrial thickness $(P<0.0001)$, menstrual cycle $(P<0.0001)$, history of ovarian surgery $(P<0.0001)$, \\ hyperandrogenism $(P<0.0001)$, and hirsutism $(P<0.0001)$ between the groups. \\ Conclusions: In general, the finding of the diminished concentration of IL-3 and IL-5 in PCOS patients may represent an altered \\ immune response to the inflammatory condition in this syndrome. \\ Keywords: Follicular fluid, Interleukin, IL-5, IL-6, Polycystic ovary syndrome
}

\section{Introduction}

Polycystic ovary syndrome (PCOS) is a multifaceted syndrome correlated to hyperandrogenism and increased renin-angiotensin system activity $(1,2)$. In other words, PCOS is a heterogeneous disease which results from interactions between genetic, epigenetic, and lifestyle factors (1). This complex endocrine disease can be observed in $5-10 \%$ of women who are in their reproductive age throughout the world $(1,2)$.

Hyperandrogenism, polycystic ovaries, and chronic anovulation based on Rotterdam criteria are the major diagnostic criteria of PCOS women (3). Further, these patients usually suffer from obesity, insulin resistance, metabolic syndrome, and cardiovascular diseases (4).

Recent research recognized PCOS as a chronic lowgrade pro-inflammatory disorder associated with different metabolic and reproductive abnormalities (5). Many circulating inflammatory markers are found to mediate PCOS in response to a stimulus, including pro-inflammatory cytokines and chemokines, as well as different endothelial inflammation markers and oxidative stress and chronic inflammation markers (6).

Cytokines involve in the regulation of ovarian function through controlling paracrine or autocrine ovarian processes (7). Furthermore, they control folliculogenesis and ovulation through providing an environment which supports follicle selection and growth. Regarding the folliculogenesis, cytokines regulate the proliferation or differentiation of cells, the survival or atresia of follicles, and oocyte maturation (8). Moreover, an impaired balance between cytokines with inflammatory and anti-inflammatory activities seems to involve in the development of PCOS (9).

T helper cell type 2 (Th2) cytokines such as interleukin (IL-3) and IL-5 contribute to inflammatory reactions and immune responses. Additionally, IL-3 secreted from helper lymphocytes, eosinophils, and natural lethal cells affects blood progesterone and mast cells $(10,11)$.

Similarly, IL-3 concentration enhances before delivery during normal pregnancy and progesterone regulates the production of this cytokine via the induction of a specific protein designated as a progesterone-induced blocking factor (12).

Likewise, IL-3 and IL-5 regulate the growth, differentiation, and activation of eosinophils under normal physiological conditions. Previous research indicated that the overexpression of IL-5 in the transgenic mice is associated with the increased number of eosinophils in

Received 14 February 2020, Accepted 29 April 2020, Available online 28 November 2020

'Department of Biology, Damghan Branch, Islamic Azad University, Damghan, Iran. ${ }^{2}$ Imam Reza Hospital, Mashhad University of Medical Science, Mashhad, Iran. ${ }^{3}$ Department of Anatomy, Mashhad Medical Sciences Branch, Islamic Azad University, Mashhad, Iran. *Corresponding Author: Vida Hojati, Tel/Fax: +98(51)38511116, Email: Vida.hojati@gmail.com 


\section{Key Messages}

- IL-6 plays an important role in maintaining the balance between Th17 cells and Treg, those effects on the development of human inflammatory diseases (31). IL-5 (a Th2 cytokine) in inflammatory diseases (PCO) decreases and tends to the immune responses are of the Th1 type (21).

female reproductive tissues (13).

In addition, IL- 6 is another cytokine that mediates inflammation and participates in the normal immune response. This pro-inflammatory cytokine increasingly expresses in female reproductive tissues. IL-6 also exhibits a crucial function in the maternal immunological tolerance to the fetus during the pregnancy (14). There are controversial results regarding the concentration of IL-6 and its association with PCOS. Some studies reported the higher expression of this cytokine in PCOS (15) while others demonstrated contrary results in this regard (16).

Based on these observations, a limited body of research is available on the effect of IL-3 and IL-5 in assisted-reproductive technology (ART) and the immunopathogenesis of PCOS. Considering controversial results on the concentration and the role of IL- 6 in this syndrome, the present study aimed to evaluate and compare the follicular fluid (FF) concentration of these cytokines in women with PCOS and healthy controls. Moreover, any possible relationship between the concentrations of mentioned cytokines and clinical factors of the patients were investigated as well.

\section{Materials and Methods}

Patient's Characteristics and Study Design

In this cross-sectional study, 39 PCOS patients in the age range of 16-40 years were randomly selected from patients referring to the in vitro fertilization (IVF) center of Imam Reza hospital (reproductive medicine unit) in Mashhad during 2018. At this center, 200-300 patients receive ART services per month. The control group included forty healthy women who referred to the IVF center for male infertility problems. An expert gynecologist diagnosed PCOS according to the Rotterdam criteria as the appearance of hyperandrogenism, oligo, or anovulation and the presence polycystic ovaries on the ultrasound scan ( $>20$ small follicles with the diameter of 2-9 $\mathrm{mm}$ and an ovarian volume of $>10 \mathrm{~mL}$ even in one ovary in the follicular phase). The exclusion criteria were receiving hormones or insulin-reducing agents during three months pre-test, the discontent of the spouse, and incomplete information, as well as adrenal dysfunction syndrome, congenital hyperplasia, autoimmune diseases, thyroid disorders, and infectious disease. The control group included fertile women who were selected from couples referring to the IVF center for men reproductive problems such as azoospermia. On the other hand, individuals with regular menstrual periods, normal range of androgen, and no history of taking medicine were included in the study as the control group. Different data were collected and compared between the groups, including demographic and clinical features of the patient and control groups comprising age, body mass index (BMI), endometrial thickness, and menstrual cycle, as well as the type of infertility and history of ovarian surgery, galactorrhea, hirsutism, dyspareunia, dysmenorrhea, and hyperandrogenism.

\section{Ovarian Stimulation Protocol}

The ovaries were stimulated using the gonadotropinreleasing hormone antagonist protocol. On the second day of the menstrual cycle, recombinant follicle-stimulating hormone treatment was initiated with a starting daily dose of 150-225 IU. A total of $0.25 \mathrm{mg}$ S.C. ovulation was triggered using 10000 IU human chorionic gonadotropin (hCG) after observing at least two follicles with a diameter of $17 \mathrm{~mm}$. Then, oocyte retrieval was carried out following 34-38 hours of hCG administration by transvaginal ultrasound-guided needle puncture. In addition, laboratory tests were performed to detect response to the stimulation, and then sonography was carried out to evaluate follicle and endometrial growth. Eventually, FF was stored inside the tubes in a sterile package first at $-20^{\circ} \mathrm{C}$ and then at $-80^{\circ} \mathrm{C}$ until IL measurements.

\section{Laboratory Tests}

IL-3 and IL-5 levels in FF samples taken from mature oocytes were measured using a sandwich ELISA kit (EASTBIOPHARM, China, Hangzhou), and the FF concentration of IL- 6 was evaluated using the human IL-6 Platinum ELISA kit (eBioscience, an Affymetrix Company, North America and Europe) according to the manufacturer's protocols.

The sensitivities of IL-3 and IL-5 assays were $1.02 \mathrm{pg} /$ $\mathrm{mL}$ and $1.52 \mathrm{ng} / \mathrm{L}$, respectively, with the intra-assay of CV $<10 \%$ and inter-assay of CV $<12 \%$ for both tests. Further, the sensitivity of IL-6 immunoassay was $0.92 \mathrm{pg} / \mathrm{m}$ with a totally intra-and inter-assay coefficient of variations as $3.4 \%$ and $5.2 \%$, respectively. Furthermore, the normal assay was in the range of 2-600 pg/ $\mathrm{mL}$ and 3-900 ng/L for IL-3 and IL-5, respectively (EASTBIOPHARM, China, Hangzhou). Eventually, IL-6 had an assay range of 1-100 $\mathrm{pg} / \mathrm{mL}$ (eBioscience, an Affymetrix Company, North America and Europe).

\section{Statistical Analysis}

All data were performed by Statistical Package software, version 16 (SPSS, Inc., Chicago, IL, USA) and descriptive statistics were expressed as the mean \pm standard deviation (SD). The normality of continuous variables was determined using a Kolmogorov-Smirnov test and the Mann-Whitney U test was performed when data did not follow a normal distribution. Moreover, a chi-square 
test was used to compare categorical variables between the groups. Finally, data were analyzed using Fisher exact test when any expected frequency was less than $20 \%$, and $P<0.05$ was considered as statistical significance.

\section{Results}

Patient's Specifications

This cross-sectional study recruited 39 infertile PCOS patients who were treated with IVF at a center in Imam Reza hospital (reproductive medicine unit) in Mashhad during 2018. The control group encompassed 34 healthy women with male infertility problems. The presence of polycystic ovaries in all PCOS patients was confirmed by ultrasound analysis and irregular periods. Based on the results, the mean age of PCOS (27.2 \pm 3.9 years) showed no significant difference with the control group $(29.0 \pm 5.3, P=0.1)$. The mean of BMI was $27.7 \pm 5.0 \mathrm{~kg} / \mathrm{m}^{2}$ and $24.8 \pm 3.4 \mathrm{~kg} /$ $\mathrm{m}^{2}$ in PCOS and control groups, respectively $(P=0.003)$. Additionally, the average endometrial thickness in PCOS patients was $8.5 \pm 1.4 \mathrm{~mm}$ whereas it was $9.9 \pm 1.08 \mathrm{~mm}$ in the healthy group $(P<0.0001)$.

The clinical features of PCOS and control groups are summarized in Table 1. As shown, a noticeable difference was observed between the two groups regarding the menstrual cycle, history of ovarian surgery, hirsutism, and hyperandrogenism $(P<0.0001$, for all variables $)$.

Follicular Fluid Concentration of ILs

The FF concentration of IL-3 in the PCOS group $(121.2 \pm 67.1 \mathrm{pg} / \mathrm{mL})$ was considerably lower than that of the control group $(188.8 \pm 120.8 \mathrm{pg} / \mathrm{mL}, P=0.004)$. Moreover, the FF concentration of IL-5 in the PCOS group $(181.5 \pm 120.8 \mathrm{pg} / \mathrm{mL})$ was lower compared to the control group $(256.6 \pm 180.2 \mathrm{pg} / \mathrm{mL}, P=0.04)$. On the other hand, IL- 6 concentration in the FF of PCOS patients $(13.1 \pm 23.7 \mathrm{pg} / \mathrm{mL})$ was higher than that of the control group $(10.5 \pm 6.7 \mathrm{pg} / \mathrm{mL})$ which did not reach a statistical significance.

Table 2 presents the relationship between clinical specifications of PCOS patients and cytokine concentrations. As shown, no significant association was found between the concentrations of studied cytokines and the clinical factors of patients.

\section{Discussion}

The present study aimed to investigate the FF concentration of interleukin (IL)-3, IL-5, and, IL-6 in infertile patients with PCOS and healthy women for controls. The results demonstrated a remarkable decline in the FF concentration of IL- 3 and IL- 5 of PCOS patients when compared to the controls.

Many researchers have studied the effect of cytokines on PCOS (17-20). As far as we know, there is no report on the assessment of IL-3 and IL-5 concentrations in the FF of PCOS patients. In the present study, the concentration of both Th2 cytokines (i.e., IL-3 and IL-5) in the FF of PCOS patients was less than that of the controls.

IL-5 is secreted by both hematopoietic (i.e., Th2, mast cells, and eosinophils) and non-hematopoietic cells in various tissues. Although in humans, IL-5 largely acts on eosinophils and their precursors, human IL-5 may involve in the production of immunoglobulin A in human mucosal tissues. Furthermore, IL- 5 stimulates the production of antibodies from activated $\mathrm{B}$ cells and contributes to the immune response, chronic inflammation, and control of disease (21). Therefore, the concentration of this cytokine increases in a normal pregnancy. In addition,

Table 1. Clinical Characteristics of the Control and PCOS

\begin{tabular}{|c|c|c|c|c|}
\hline Variables & & $\operatorname{PCOS}(n=39)$ & Control $(n=34)$ & $P$ Value \\
\hline \multirow{2}{*}{ Menstrual cycle } & Regular & $24(61.5 \%)$ & $24(61.5 \%)$ & \multirow{2}{*}{$<0.0001^{\mathrm{a}^{*}}$} \\
\hline & Irregular & $15(38.5 \%)$ & $15(38.5 \%)$ & \\
\hline \multirow{2}{*}{ Surgical history } & Negative & $22(56.4 \%)$ & $22(56.4 \%)$ & \multirow{2}{*}{$<0.0001^{b^{*}}$} \\
\hline & Positive & $17(43.6 \%)$ & $17(43.6 \%)$ & \\
\hline \multirow{2}{*}{ Type of infertility } & Primary & $35(89.7 \%)$ & $35(89.7 \%)$ & \multirow{2}{*}{$0.263^{\mathrm{a}}$} \\
\hline & Secondary & $4(10.3 \%)$ & $4(10.3 \%)$ & \\
\hline \multirow{2}{*}{ Galactorrhea } & Negative & $38(97.4 \%)$ & $38(97.4 \%)$ & \multirow{2}{*}{$1.00^{\mathrm{a}}$} \\
\hline & Positive & $1(2.6 \%)$ & $1(2.6 \%)$ & \\
\hline \multirow{2}{*}{ Hirsutism } & Negative & $24(61.5 \%)$ & $24(61.5 \%)$ & \multirow{2}{*}{$<0.0001^{b^{*}}$} \\
\hline & Positive & $15(38.5 \%)$ & $15(38.5 \%)$ & \\
\hline \multirow{2}{*}{ Dysparony } & Negative & $37(94.9 \%)$ & $37(94.9 \%)$ & \multirow{2}{*}{$0.495^{\mathrm{a}}$} \\
\hline & Positive & $2(5.1 \%)$ & $2(5.1 \%)$ & \\
\hline \multirow{2}{*}{ Dysmenorrhea } & Negative & $33(84.6 \%)$ & $33(84.6 \%)$ & \multirow{2}{*}{$1.00^{\mathrm{b}}$} \\
\hline & Positive & $6(15.4 \%)$ & $6(15.4 \%)$ & \\
\hline \multirow{2}{*}{ Hyperandrogenism } & Negative & $7(17.9 \%)$ & $7(17.9 \%)$ & \multirow{2}{*}{$<0.0001^{b^{*}}$} \\
\hline & Positive & $32(82.1 \%)$ & $32(82.1 \%)$ & \\
\hline
\end{tabular}

Note. SD, Standard deviation; PCOS, Polycystic ovary syndrome; Data are presented as mean \pm SD.

a Based on Fisher's exact test; ${ }^{\text {b }}$ Based on the chi-square test; ${ }^{*} P$ value less than $0.05 ;{ }^{* *} P$ value less than 0.01 . 
Table 2. Relationship Between the Clinical Characteristics of Polycystic Ovary Syndrome Patients and Cytokine Concentration

\begin{tabular}{|c|c|c|c|c|c|c|c|}
\hline \multirow{2}{*}{ Variables } & & \multicolumn{2}{|c|}{ Interleukin 3} & \multicolumn{2}{|c|}{ Interleukin 5} & \multicolumn{2}{|c|}{ Interleukin 6} \\
\hline & & Mean \pm SD & $P$ Value $^{\mathrm{a}}$ & Mean \pm SD & $P$ Value $^{\mathrm{a}}$ & Mean \pm SD & $P$ Value $^{\mathrm{a}}$ \\
\hline \multirow{2}{*}{ Menstrual cycle } & Regular & $155.0 \pm 99.1$ & \multirow{2}{*}{0.323} & $214.8 \pm 156.8$ & \multirow{2}{*}{0.223} & $12.5 \pm 19.8$ & \multirow{2}{*}{0.323} \\
\hline & Irregular & $143.6 \pm 88.03$ & & $222.9 \pm 152.7$ & & $9.6 \pm 6.1$ & \\
\hline \multirow{2}{*}{ Surgical history } & Negative & $169.6 \pm 102.8$ & \multirow{2}{*}{0.098} & $235.3 \pm 163.9$ & \multirow{2}{*}{0.347} & $9.6 \pm 6.3$ & \multirow{2}{*}{0.967} \\
\hline & Positive & $97.1 \pm 35.5$ & & $154.4 \pm 101.9$ & & $19.334 .9 \pm$ & \\
\hline \multirow{2}{*}{ Type of infertility } & Primary & $157.4 \pm 98.3$ & \multirow{2}{*}{0.567} & $221.3 \pm 158.8$ & \multirow{2}{*}{0.608} & $12.4 \pm 18.4$ & \multirow{2}{*}{0.608} \\
\hline & Secondary & $88.9 \pm 10.4$ & & $150.06 \pm 60.1$ & & $5.1 \pm 1.9$ & \\
\hline \multirow{2}{*}{ Galactorrhea } & Negative & $153.4 \pm 96.9$ & \multirow{2}{*}{0.821} & $219.4 \pm 153.9$ & \multirow{2}{*}{0.06} & $11.9 \pm 17.9$ & \multirow{2}{*}{0.462} \\
\hline & Positive & 103.2 & & 5.00 & & 11.4 & \\
\hline \multirow{2}{*}{ Hirsutism } & Negative & $158.9 \pm 99.4$ & \multirow{2}{*}{0.658} & $231.8 \pm 155.4$ & \multirow{2}{*}{0.131} & $12.7 \pm 19.9$ & \multirow{2}{*}{0.323} \\
\hline & Positive & $128.5 \pm 82.5$ & & $157.2 \pm 142.8$ & & $8.7 \pm 4.8$ & \\
\hline \multirow{2}{*}{ Disparony } & Negative & $152.1 \pm 97.7$ & \multirow{2}{*}{0.113} & $214.6 \pm 155.9$ & \multirow{2}{*}{0.194} & $11.9 \pm 18.1$ & \multirow{2}{*}{0.567} \\
\hline & Positive & $172.8 \pm 28.5$ & & $282.8 \pm 130.4$ & & $9.4 \pm 1.5$ & \\
\hline \multirow{2}{*}{ Dysmenorrhea } & Negative & $142.3 \pm 90.4$ & \multirow{2}{*}{0.33} & $197.8 \pm 149.8$ & \multirow{2}{*}{0.085} & $12.5 \pm 19.3$ & \multirow{2}{*}{0.829} \\
\hline & Positive & $211.1 \pm 112.5$ & & $321.5 \pm 147.4$ & & $8.5 \pm 4.5$ & \\
\hline \multirow{2}{*}{ Hyperandrogenism } & Negative & $172.1 \pm 108.6$ & \multirow{2}{*}{0.154} & $232.5 \pm 174.8$ & \multirow{2}{*}{0.892} & $9.9 \pm 6.6$ & \multirow{2}{*}{0.559} \\
\hline & Positive & $127.9 \pm 72.5$ & & $195.9 \pm 124.7$ & & $14.4 \pm 25.9$ & \\
\hline
\end{tabular}

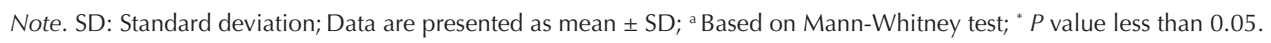

the decreased concentration of IL-5 in PCOS patients indicates alternations in the FF levels of this antiinflammatory cytokine.

IL-3 and IL-5, as the members of the $\beta$ c receptor family, have a beta-receptor subunit $(\beta c)$ and show similar biological activities. Further, aberrant signaling through $\beta c$ can lead to chronic inflammatory conditions such as PCOS (22).

A reduction in IL-3 and IL-5 levels as a suppressor factor for the inflammation in the PCOS probably represents a decline in $\mathrm{T}$ helper (Th2)-related cytokines and a bias toward Th1-type immune responses. This assertion is in line with the findings of previous studies, indicating a higher concentration of Th1-related cytokines and an increased ratio of Th1/Th2 in PCOS patients compared to women with normal ovarian function (23).

There are arguably results regarding the association between IL-6 levels and PCOS $(3,15)$. According to (24), IL-6 performs a crucial character in keeping the balance between Th17 cells and regulatory T cells (Treg). Furthermore, Th17 cells are a subset of pro-inflammatory cells while Treg cells represent an antagonistic effect. Moreover, their growth pathways are cross-linked and there is significant flexibility between these cells. Additionally, the balance between Th17 and Treg cells determines the development and outcome of autoimmune/inflammatory diseases, and impaired balance can aggravate inflammation (25). In PCOS patients, IL-6 might apparently induce Th17 cells, mediating inflammation and tissue damage (26). In the current study, the FF concentration IL-6 in PCOS patients was higher compared to the healthy group although the difference was not statistically notable. The findings of a study also demonstrated a higher serum and
FF concentration of IL-6 in PCOS patients (27) while those of another one represented no significant association in this regard (6). These discrepancies can be related to the difference in the sample size, diverse studied populations, different inclusion criteria, and analysis methods. A recent systematic review and meta-analysis indicated that higher IL-6 concentrations in PCOS patients are associated with insulin resistance and androgen levels. Therefore, the concentration of IL- 6 for monitoring the treatment of these patients can be used as a biomarker but not as a diagnostic tool (3).

Although Th2 cells produce IL-6, this anti-inflammatory cytokine can be secreted from other cells such as Th1 and B cells and mediate the host's response to inflammation and infection conditions (28). Moreover, IL-6 concentration increases relatively in the placenta, amniotic fluid, and choriodecidua from women with preterm labor compared to those with term delivery. Similarly, the enhanced level of IL-6 is one of the most sensitive and specific indices of preterm delivery associated with infections (29). It seems that the FF concentration of this anti-inflammatory cytokine reduces during normal pregnancy. The results of this study revealed a non-significant elevation in the FF of pro-inflammatory cytokine in PCOS patients probably due to the sample size of the studied population.

Furthermore, the results of the present study indicated that women with irregular menstrual cycles for developing PCOS are at a higher risk. The confusion PCOS disturbs a lady's endocrine framework and expands the circulating levels of free testosterone as a marker for hyperandrogenism, which causes hirsutism in women (30). Eventually, testosterone levels in PCOS patients were meaningfully higher than those of the healthy group. 


\section{Conclusions}

In summary, it seems that the altered immune response to the inflammatory status in the PCOS could be related to the diminished concentration of IL- 3 and IL-5 as Th2 cytokines. However, the FF concentration of IL- 6 in PCOS patients was not statistically significant probably because of the low sample size of the study society. Accordingly, further investigations with larger sample sizes and the use of molecular techniques are recommended for clarifying the link between pro- and anti-inflammatory factors and PCOS pathogenesis and providing a new diagnostic tool in these patients undergoing assisted reproductive technology.

\section{Authors' Contribution}

MG: Manuscript drafting; $\mathrm{VH}$ and BS: Critical revision of the manuscript for important intellectual content and statistical analysis; AA: Study concept and design, as well as data analysis and interpretation.

\section{Conflict of Interests}

Authors declare that they have no conflict of interests.

\section{Ethical Issues}

This study was approved by the Bioethics Research Committee, Islamic Azad University, Damghan Branch (IR.IAU.REC 14230525981001). Additionally, informed consent forms were taken from all participants.

\section{Financial Support}

None.

\section{Acknowledgments}

The authors wish to thank the staff of Milad Infertility and Research Center, Imam Reza Hospital, Mashhad University of Medical Sciences and Islamic Azad University of Damghan for their kind assistance.

\section{References}

1. Rashid N, Nigam A, Saxena P, Jain SK, Wajid S. Association of IL-1 $\beta$, IL-1Ra and FABP1 gene polymorphisms with the metabolic features of polycystic ovary syndrome. Inflamm Res. 2017;66(7):621-636. doi:10.1007/s00011-017-1045-3

2. Foroozanfard F, Soleimani A, Arbab E, Samimi M, Tamadon MR. Relationship between IL-17 serum level and ambulatory blood pressure in women with polycystic ovary syndrome. J Nephropathol. 2017;6(1):15-24. doi:10.15171/jnp.2017.04

3. Peng Z, Sun Y, Lv X, Zhang H, Liu C, Dai S. Interleukin-6 levels in women with polycystic ovary syndrome: a systematic review and meta-analysis. PLoS One. 2016;11(2):e0148531. doi:10.1371/journal.pone.0148531

4. Yildizhan B, Anik Ilhan G, Pekin T. The impact of insulin resistance on clinical, hormonal and metabolic parameters in lean women with polycystic ovary syndrome. J Obstet Gynaecol. 2016;36(7):893-896. doi:10.3109/01443615.201 6.1168376

5. Shorakae S, Teede H, de Courten B, Lambert G, Boyle J, Moran LJ. The emerging role of chronic low-grade inflammation in the pathophysiology of polycystic ovary syndrome. Semin Reprod Med. 2015;33(4):257-269. doi:10.1055/s-0035-1556568

6. Escobar-Morreale HF, Luque-Ramírez M, González F. Circulating inflammatory markers in polycystic ovary syndrome: a systematic review and metaanalysis. Fertil Steril. 2011;95(3):1048-1058.e1041-1042. doi:10.1016/j. fertnstert.2010.11.036

7. Tesone M, Abramovich D, Irusta G, Parborell F. Autocrine and paracrine regulation of the ovary. In: Chedrese P, ed. Reproductive Endocrinology. Boston, MA: Springer; 2009:241248. doi:10.1007/978-0-387-88186-7_21.241-8

8. Field SL, Dasgupta T, Cummings M, Orsi NM. Cytokines in ovarian folliculogenesis, oocyte maturation and luteinisation. Mol Reprod Dev. 2014;81(4):284-314. doi:10.1002/ mrd.22285

9. Ebejer K, Calleja-Agius J. The role of cytokines in polycystic ovarian syndrome. Gynecol Endocrinol. 2013;29(6):536-540. doi:10.3109/09513590.2012.760195

10. Kritas SK, Saggini A, Cerulli G, et al. Interrelationship between IL-3 and mast cells. J Biol Regul Homeost Agents. 2014;28(1):17-21.

11. Kouro T, Takatsu K. IL-5- and eosinophil-mediated inflammation: from discovery to therapy. Int Immunol. 2009;21(12):1303-1309. doi:10.1093/intimm/dxp102

12. Szekeres-Bartho J, Šućurović S, Mulac-Jeričević B. The role of extracellular vesicles and PIBF in embryo-maternal immuneinteractions. Front Immunol. 2018;9:2890. doi:10.3389/ fimmu.2018.02890

13. Sferruzzi-Perri AN, Robertson SA, Dent LA. Interleukin-5 transgene expression and eosinophilia are associated with retarded mammary gland development in mice. Biol Reprod. 2003;69(1):224-233. doi:10.1095/biolreprod.102.010611

14. Prins JR, Gomez-Lopez N, Robertson SA. Interleukin-6 in pregnancy and gestational disorders. J Reprod Immunol. 2012;95(1-2):1-14. doi:10.1016/j.jri.2012.05.004

15. Küçük $M$, Altınkaya $S$, Nergiz $S$, et al. Interleukin-6 levels in relation with hormonal and metabolic profile in patients with polycystic ovary syndrome. Gynecol Endocrinol. 2014;30(6):423-427. doi:10.3109/09513590.2014.895981

16. Jatzko B, Ott J. Circulating inflammatory markers in polycystic ovary syndrome: a systematic review and meta-analysis. Fertil Steril. 2011;96(4):e158. doi:10.1016/j.fertnstert.2011.07.1098

17. Daan NM, Koster MP, de Wilde MA, et al. Biomarker profiles in women with PCOS and PCOS offspring; a pilot study. PLoS One. 2016;11(11):e0165033. doi:10.1371/journal. pone. 0165033

18. Sykes L, Maclntyre DA, Yap XJ, Ponnampalam S, Teoh TG, Bennett PR. Changes in the Th1:Th2 cytokine bias in pregnancy and the effects of the anti-inflammatory cyclopentenone prostaglandin 15-deoxy-Delta(12,14)prostaglandin J2. Mediators Inflamm. 2012;2012:416739. doi: 10.1155/2012/416739

19. Sykes L, Macintyre DA, Yap XJ, Teoh TG, Bennett PR. The Th1:th2 dichotomy of pregnancy and preterm labour. Mediators Inflamm. 2012;2012:967629. doi: 10.1155/2012/967629

20. Uslu B, Johnson J. The In Vitro culture of ovarian follicles: A brief history and current considerations. In: Krisher RL, ed. Oocyte Physiology and Development in Domestic Animals. 1st ed. John Wiley and Sons, Inc; 2013:21-34.

21. Takatsu K. Interleukin-5 and IL-5 receptor in health and diseases. Proc Jpn Acad Ser B Phys Biol Sci. 2011;87(8):463485. doi:10.2183/pjab.87.463

22. Broughton $\mathrm{SE}, \mathrm{Nero} T \mathrm{TL}$, Dhagat $\mathrm{U}$, et al. The $\beta$ c receptor family Structural insights and their functional implications. Cytokine. 2015;74(2):247-258. doi:10.1016/j.cyto.2015.02.005

23. Qin L, Xu W, Li X, et al. Differential expression profile of immunological cytokines in local ovary in patients with polycystic ovarian syndrome: analysis by flow cytometry. Eur J Obstet Gynecol Reprod Biol. 2016;197:136-141. doi:10.1016/j.ejogrb.2015.12.003 
24. Lee GR. The Balance of Th17 versus Treg Cells in Autoimmunity. Int J Mol Sci 2018; 19. doi: 10.3390/ijms19030730

25. Noack M, Miossec P. Th17 and regulatory T cell balance in autoimmune and inflammatory diseases. Autoimmun Rev. 2014;13(6):668-677. doi:10.1016/j.autrev.2013.12.004

26. Nasri F, Doroudchi M, Namavar Jahromi B, Gharesi-Fard B. T helper cells profile and CD4+CD25+Foxp3+regulatory T cells in polycystic ovary syndrome. Iran J Immunol. 2018;15(3):175185. doi:10.22034/iji.2018.39387

27. Artimani T, Karimi J, Mehdizadeh M, et al. Evaluation of prooxidant-antioxidant balance (PAB) and its association with inflammatory cytokines in polycystic ovary syndrome (PCOS). Gynecol Endocrinol. 2018;34(2):148-152. doi:10.1080/0951 3590.2017 .1371691
28. $\mathrm{Na} \mathrm{H}$, Cho M, Chung Y. Regulation of Th2 cell immunity by dendritic cells. Immune Netw. 2016;16(1):1-12. doi:10.4110/ in.2016.16.1.1

29. Sykes L, Maclntyre DA, Yap XJ, Teoh TG, Bennett PR. The Th1:th2 dichotomy of pregnancy and preterm labour. Mediators Inflamm. 2012;2012:967629. doi:10.1155/2012/967629

30. Rosenfield RL, Ehrmann DA. The pathogenesis of polycystic ovary syndrome (PCOS): the hypothesis of PCOS as functional ovarian hyperandrogenism revisited. Endocr Rev. 2016;37(5):467-520. doi:10.1210/er.2015-1104

31. Wei M, Cheng Y, Bu H, Zhao Y, Zhao W. Length of Menstrual Cycle and Risk of Endometriosis: A Meta-Analysis of 11 CaseControl Studies. Medicine (Baltimore) 2016;95:e2922. doi: 10.1097/MD.0000000000002922

(C) 2021 The Author(s); This is an open-access article distributed under the terms of the Creative Commons Attribution License (http:// creativecommons.org/licenses/by/4.0), which permits unrestricted use, distribution, and reproduction in any medium, provided the original work is properly cited. 